\title{
Analytical modelling of the three-dimensional steady-state temperature in a bearing ring
}

\author{
Mohamed Hamraoui ${ }^{1, a}$, Talaat Osman $^{2}$, Abderrahmane Boucheffa $^{2}$ \\ and Mohammad Mehdi Rashidi ${ }^{3}$ \\ 1 Université Hassan II Aïn chock, École Supérieure de Technologie de Casablanca, Laboratoire RITM, Km 7, \\ Route El Jadida, BP 8012 Oasis, Casablanca, Morocco \\ 2 Université Paris Sud, IUT d'Orsay, Département MP, Plateau du Moulon, 91400 Orsay, France \\ 3 Mechanical Engineering Department, Engineering Faculty of Bu-Ali Sina University, Hamedan, Iran
}

Received 11 October 2010, Accepted 30 December 2010

\begin{abstract}
An analytical solution to compute the 3D steady state temperature distribution in a bearing ring is presented in this paper. The ring is formed by a hollow cylinder of finite length. Its radial external surface is subjected to localized, identical heat sources, equally spaced in the azimuth direction. This surface is also subjected to convective cooling while as the internal surface is maintained at a uniform temperature. The developed solution is explicit and does not impose any restriction on the geometrical or physical parameters.
\end{abstract}

Key words: Heat conduction / analytical solution / bearing / tribology / interfaces / moving heat sources

\section{Introduction}

Bearings are used in many industrial sectors, usually to ensure the mechanical guidance of rotating pieces. Among these industrial applications are: (i) motor gearboxes, (ii) wheels of land vehicles, (iii) turbo-pumps of engine space vehicles, (iv) all mechanics systems involving rotating motion. The diameters of the bearing are of the order of few centimeters and the rotating velocity can vary from a few thousands rpm (common areas) to a few tens of thousands of rpm (space area). The mechanical charge, in addition to the relative motion between the ball-bearing and the ring produce a heat flux at the contact levels. The dissipated heat leads to mechanical stresses, material wear and fatigue (due to the periodic thermal solicitation). Therefore, it is important to provide a computational algorithm to evaluate the temperature distribution in such systems for given operating conditions.

Many works are developed in the literature to evaluate the temperature in a solid subjected to frictional heating in a general way [1-10], or in the particular case of ball-bearings [11]. The difficulty in such problems resides in the presence of a relative motion in addition to

\footnotetext{
${ }^{a}$ Corresponding author: hamraoui@est-uh2c.ac.ma
}

\section{Nomenclature}

\begin{tabular}{|ll|}
\hline$c$ & Semi-axial dimension of the contact area, $\mathrm{m}$ \\
$C$ & Semi-axial dimension of the ring, $\mathrm{m}$ \\
$h$ & Heat transfer coefficient, W. $\mathrm{m}^{-2} \cdot \mathrm{K}^{-1}$ \\
$q$ & Heat flux density, W.m ${ }^{-2}$ \\
$R_{0}$ & Inner radius or the ring, $\mathrm{m}$ \\
$R_{1}$ & Outer radius or the ring, $\mathrm{m}$ \\
$r, \theta, z$ & Polar coordinates \\
$T$ & Temperature (relative to ambient), $\mathrm{K}$ \\
$\mathrm{Greek}$ & symbols \\
$\alpha$ & Thermal diffusivity, $\mathrm{m}^{2} \cdot \mathrm{s}^{-1}$ \\
$\lambda$ & Thermal conductivity, W. $\mathrm{m}^{-1} \cdot \mathrm{K}^{-1}$ \\
$\beta$ & Semi-angle of the contact area, rad \\
$\theta_{0}$ & Semi-angle of a sector, rad \\
$\omega$ & Angular velocity, $\mathrm{s}^{-1}$ \\
\hline
\end{tabular}

the very small contact areas between the concerned solids. Interface heat transfer problems arise in such situations. Some works are devoted to deal with this aspect [12-15]. However, many questions need to be clarified, particularly those who are related to the real contact areas and their evolution during a friction process. This paper is essentially concerned with the local temperature evaluation in the case of known contact area and specified heat 
flux. The problem of an internal ring, subjected to localised identical heat sources, equally spaced in the azimuth direction is treated here. The external ring may be treated using the same formulation. The heat sources simulate the contact areas between the rotating ring and the cylindrical rollers considered here.

In what follows, we describe the problem and give the details of the proposed solution in addition to some applied examples.

\section{Mathematical model}

\subsection{Description of the problem}

This study is concerned with the interior ring (of a ball-bearing) with internal and external radius, respectively, $R_{0}$ and $R_{1}$. The external surface of the ring, whose depth is $2 C$, is subjected to identical heat sources of depth $2 C$ through an angular aperture of $2 \beta$. This surface is cooled by convection of coefficient $h$ and with zero ambient temperature.

The interior surface is maintained at a zero reference temperature (which simulates the contact with a rod of high thermal capacity).

The heat flux received by the ring is considered to be uniform, of density $q$. The angular rotational velocity of the ring is $\omega$. The heat losses across the relatively small lateral surfaces are negligible. Taking the angular periodicity and the axial symmetry into consideration the calculation can be limited to an elementary cell of depth $C$ and an angular aperture $2 \theta_{0}$ as shown in Figure 1.

\subsection{Governing equations}

The thermal conductivity $\lambda$ as well as the thermal diffusivity $\alpha$ of the material are considered to be independent of the temperature. The steady state heat diffusion equation in a three-dimensional coordinate, relative to the heat source is given by:

$$
\frac{\partial^{2} T}{\partial r^{2}}+\frac{1}{r} \frac{\partial T}{\partial r}+\frac{1}{r^{2}} \frac{\partial^{2} T}{\partial \theta^{2}}-\frac{\omega}{\alpha} \frac{\partial T}{\partial \theta}+\frac{\partial^{2} T}{\partial z^{2}}=0
$$

with the periodicity conditions:

$$
T\left(r,-\theta_{0}, z\right)=T\left(r, \theta_{0}, z\right) ; \frac{\partial T\left(r,-\theta_{0}, z\right)}{\partial \theta}=\frac{\partial T\left(r, \theta_{0}, z\right)}{\partial \theta}
$$

and the boundary conditions:

$$
\begin{gathered}
\frac{\partial T(r, \theta, 0)}{\partial z}=0 ; \frac{\partial T(r, \theta, C)}{\partial z}=0 \\
\lambda \frac{\partial T\left(R_{1}, \theta, z\right)}{\partial r}=\mid \begin{array}{r}
q:(-\beta \leq \theta \leq \beta, 0 \leq z \leq c) \\
-h T\left(R_{1}, \theta, z\right):\left(-\theta_{0} \leq \theta \leq \theta_{0}\right. \\
0 \leq z \leq C) \\
T\left(R_{0}, \theta, z\right)=0
\end{array}
\end{gathered}
$$

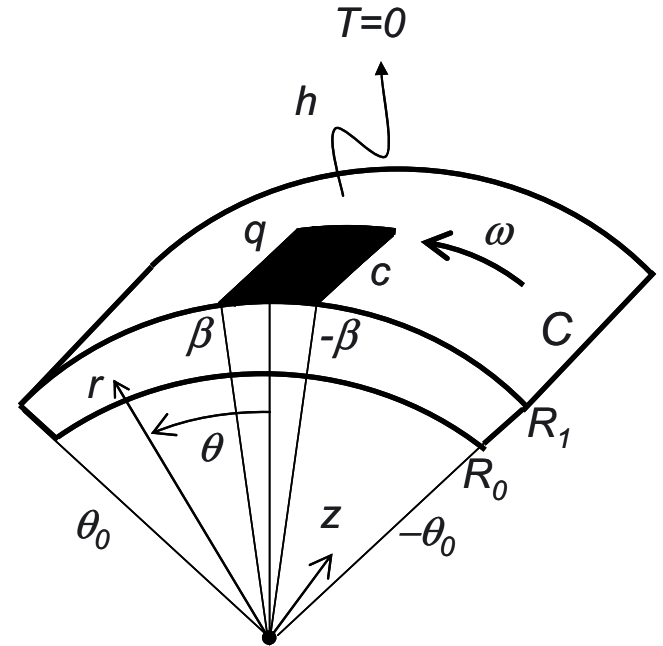

Fig. 1. Elementary cell of a bearing ring.

The heat transfer coefficient $h$ incorporates the convective and radiative exchanges. The radiative exchanges in a bearing are negligible because the surfaces of rings and balls are at close temperatures. The heat convection coefficient $h$ can be evaluated by using the correlations for cavities [16].

\subsection{Analytical solution}

The periodicity condition (with respect to $\theta$ ) as well the axial adiabacity and symmetry condition (in the $z$ direction) allow to use the following integral transforms [2]:

$$
\begin{aligned}
T_{m}^{*} & =\frac{1}{2 \theta_{0}} \int_{-\theta_{0}}^{\theta_{0}} T \exp \left(-j m \pi \theta / \theta_{0}\right) \mathrm{d} \theta ; \\
T_{m n}^{* *} & =\frac{1}{C} \int_{0}^{C} T_{m}^{*} \cos (n \pi z / C) \mathrm{d} z
\end{aligned}
$$

where $j$ is the imaginary number.

The double transformation of $T$ yields the following equations:

$$
\begin{aligned}
& \frac{\mathrm{d}^{2} T_{m n}^{* *}}{\mathrm{~d} r^{2}}+\frac{1}{r} \frac{\mathrm{d} T_{m n}^{* *}}{\mathrm{~d} r}-\left[\frac{\left(m \pi / \theta_{0}\right)^{2}}{r^{2}}+\left(\frac{n \pi}{C}\right)^{2}+j \frac{m \pi \omega}{\theta_{0} \alpha}\right] \\
& \times T_{m n}^{* *}=0 \\
& \lambda \frac{\mathrm{d} T_{m n}^{* *}\left(R_{1}\right)}{\mathrm{d} r}=q_{m n}^{* *}-h T_{m n}^{* *}\left(R_{1}\right) ; T_{m n}^{* *}\left(R_{0}\right)=0
\end{aligned}
$$

with:

$$
\begin{aligned}
q_{m n}^{* *} & =q \frac{\sin \left(m \pi \beta / \theta_{0}\right) \sin (n \pi c / C)}{(m \pi)(n \pi)} ; \quad q_{00}^{* *}=q \frac{\beta c}{\theta_{0} C} \\
q_{0 n}^{* *} & =\frac{q \beta}{\theta_{0}} \frac{\sin (n \pi c / C)}{(n \pi)} ; \quad q_{m 0}^{* *}=\frac{q c}{C} \frac{\sin \left(m \pi \beta / \theta_{0}\right)}{(m \pi)}
\end{aligned}
$$


Table 1. Numerical data.

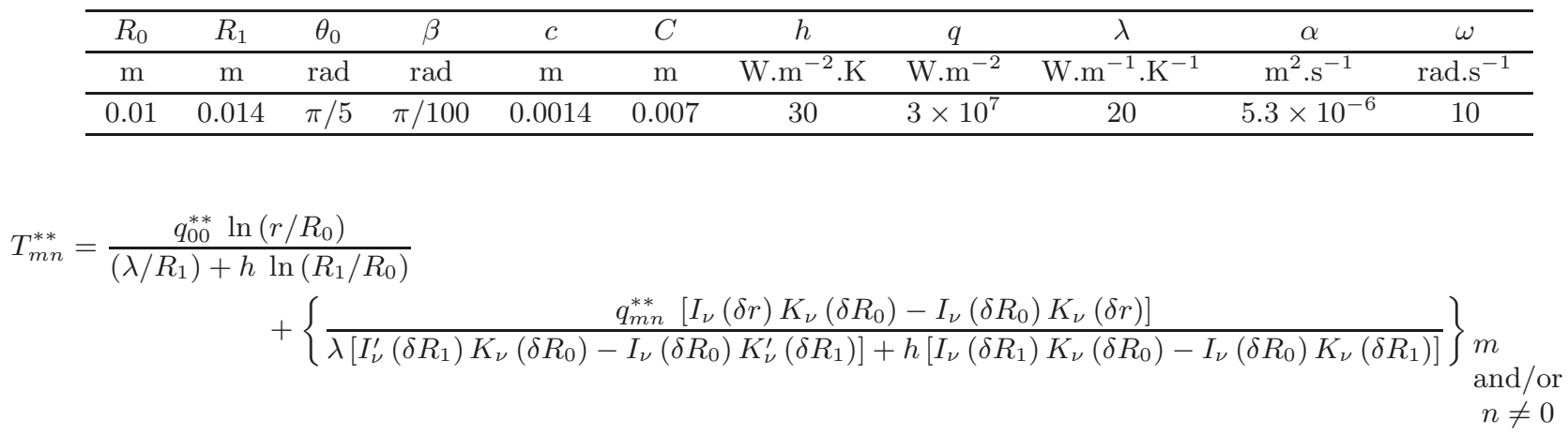

The solution of Equation (1a) with the boundary conditions (4a) can be written as follows

See equation (7) above.

with:

$$
\nu=\frac{m \pi}{\theta_{0}}, \quad \delta=\left[\left(\frac{n \pi}{C}\right)^{2}+j \frac{m \pi \omega}{\theta_{0} \alpha}\right]^{1 / 2}
$$

$I_{\nu}$ and $K_{\nu}$ are, respectively, the modified Bessel functions of the first and second kinds of order $\nu$.

$I_{\nu}^{\prime}$ and $K_{\nu}^{\prime}$ are their respective derivatives, given by :

$$
\begin{aligned}
I_{\nu}^{\prime}\left(\delta R_{1}\right) & =I_{\nu}\left(\delta R_{1}\right) / R_{1}+\delta I_{\nu+1}\left(\delta R_{1}\right) \\
K_{\nu}^{\prime}\left(\delta R_{1}\right) & =K_{\nu}\left(\delta R_{1}\right) / R_{1}-\delta K_{\nu+1}\left(\delta R_{1}\right)
\end{aligned}
$$

Using the inverse transforms of (5)

$$
\begin{aligned}
T & =\sum_{m=0}^{\infty} \varepsilon_{m} \Re\left[T_{m}^{*} \exp \left(j m \pi \theta / \theta_{0}\right)\right] ; \\
T_{m}^{*} & =\sum_{n=0}^{\infty} \varepsilon_{n} T_{m n}^{* *} \cos (n \pi z / C)
\end{aligned}
$$

with $\varepsilon_{0}=1$ and $\varepsilon_{k \neq 0}=2$

Where $\Re[$.$] is the real part of the term between brack-$ ets, we obtain the following expression for the threedimensional temperature distribution in the solid:

$$
\begin{aligned}
& T(r, \theta, z)=\frac{q_{00}^{* *} \ln \left(r / R_{0}\right)}{\left(\lambda / R_{1}\right)+h \ln \left(R_{1} / R_{0}\right)} \\
& +2 \sum_{n=1}^{\infty} T_{0 n}^{* *} \cos \left(\frac{n \pi z}{C}\right)+2 \sum_{m=1}^{\infty} \Re\left[T_{m 0}^{* *} \exp \left(j m \pi \theta / \theta_{0}\right)\right] \\
& \quad+4 \sum_{n=1}^{\infty} \cos \left(\frac{n \pi z}{C}\right) \sum_{m=1}^{\infty} \Re\left[T_{m n}^{* *} \exp \left(j m \pi \theta / \theta_{0}\right)\right]
\end{aligned}
$$

\section{Results and analysis}

The explicit solution given by Equation (9) is used here to compute the three dimensional temperature field in the internal ring of a ball-bearing using the data shown

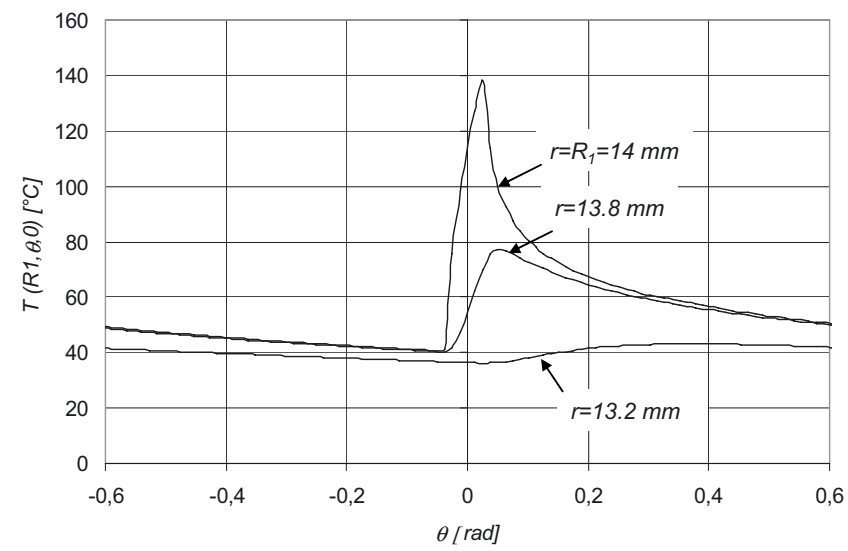

Fig. 2. Ring temperature at different radii $(z=0)$.

in Table 1. The number of terms necessary to ensure convergence of the series depends on geometric ratio $c / C$ and $\beta / \theta_{0}$. The number of terms is great when the geometrical ratio is small. Typically, it should take about 100 terms for ratios of 0.1 and 1000 terms for ratios of 0.01 .

Figure 2 shows the temperature variation with the angular position for three different radii. It can be seen that the temperature values are close from each other in the regions situated before and after the contact zone, in which a relativity sensible differences between them are observed. The strong thermal gradients in the contact zone lead to residual stresses and hence to a cyclic fatigue in the ring.

However, these gradients appear only in a zone relatively close to the external surface (i.e. for $r$ near to $R_{1}$ ). Then, the temperatures become almost independent of the angular position for lower $r$ values (e.g. $r=13.2 \mathrm{~mm}$ ).

Figure 3 shows the temperature variation with the normalized ring radius below the contact area $(\theta=0$, $z=0)$. A very strong temperature drop can be observed in a narrow zone near the external surface, followed by a progressive decrease down to the internal one (which is supposed to be at the zero reference temperature). This variation follows a logarithmic law, represented by the first term of Equation (9). 


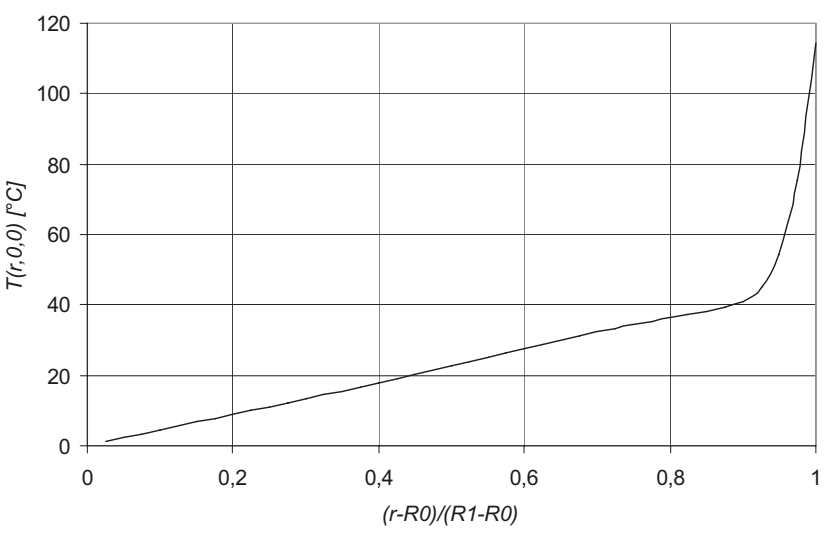

Fig. 3. Ring temperature as a function of a radius $(\theta=0$, $z=0)$.

These results show that only a narrow zone, near the external surface, is subjected to a cyclic temperatures variation. Beyond this region, a nearly one-dimensional heat diffusion may be considered.

\section{Conclusion}

An exact analytical model to compute the steady state, three-dimensional temperature field in a ball- bearing ring, is presented in this paper. The solution is explicit, easy to program and does not impose any restriction on the system parameters. In this solution, the difficulties related to a numerical model - due to the relative motion and to ring heat sources - are avoided.

\section{References}

[1] T. Osman, A. Boucheffa, Analytical solution for the 3D steady state conduction in a solid subjected to a moving rectangular heat source and surface cooling, C. R. Mécanique 337 (2009) 107-111

[2] N. Laraqi, Thermal constriction phenomenon in sliding contacts, Int. J. Heat Mass Trans. 39 (1996) 3717-3724
[3] N.R. DesRuisseaux, R.D. Zerkle, Temperature in semiinfinite and cylindrical bodies subject to moving heat sources and surface cooling, ASME J. Heat Transf. (1970) 456-464

[4] M. Hamraoui, Z. Zouaoui, Modelling of heat transfer between two rollers in dry friction, Int. J. Therm. Sci. 48 (2009) 1243-1246

[5] J.C. Jaeger, Moving sources of heat and the temperature at sliding contacts, Proc. Royal Soc. NSW 76 (1942) 203224

[6] A. Baïri, J.M. Garcia-de-Maria, N. Laraqi, Effect of thickness and thermal properties of film on the thermal behavior of moving rough interfaces, EpJ AP 26 (2004) 29-34

[7] M. Hamraoui, Thermal behaviour of rollers during the rolling process, Appl. Therm. Eng. 29 (2009) 2386-2390

[8] N. Laraqi, N. Alilat, J.M. Garcia-de-Maria, A. Baïri, Temperature and division of heat in a pin-on-disc frictional device - Exact analytical solution, Wear 266 (2009) $765-770$

[9] B. Gecim, W.O.Winer, Steady temperature in a rotating cylinder subject to surface heating and convective cooling, ASME J. Trib. 106 (1984) 120-127

[10] A. Baïri, N. Alilat, J.G. Bauzin, N. Laraqi, Threedimensional stationary thermal behavior of a bearing ball, Int. J. Therm. Sci. 43 (2004) 561-568

[11] F.F. Ling and T.E. Simkins, Measurement of point-wise juncture condition of temperature at the interface of two bodies in sliding contact, ASME J. Basic Eng. 85 (1963) 481-486

[12] N. Laraqi, An exact explicit analytical solution of the steady-state temperature in a half space subjected to a moving circular heat source, J. Tribol. 125 (2003) 859-862

[13] J.P. Bardon, Bases physiques des conditions de contact thermique imparfait entre milieux en glissement relatif, Revue Générale de Thermique Fr. 386 (1994) 86-91

[14] N. Laraqi, Thermal impedance and transient temperature due to a spot of heat on a half-space, Int. J. Therm. Sci. 49 (2010) 529-533

[15] M. Amara, V. Timchenko, M. El Ganaoui, E. Leonardi, G. de Vahl Davis, A 3D computational model of heat transfer coupled to phase change in multilayer materials with random thermal contact resistance, Int. J. Therm. Sci. 48 (2009) 421-427

[16] A. Baïri, N. Laraqi, J.M. Garcia de Maria, Numerical and experimental study of natural convection in tilted parallelepipedic cavities for large Rayleigh numbers, Exp. Therm. Fluid Sci. 31 (2007) 309-324 